\title{
MAC PROTOCOLS FOR COOPERATIVE DIVERSITY IN WLAN
}

\author{
Unnikrishnan $\mathbf{M}^{1}$, Rinu Titus ${ }^{2}$, Premkumar $\mathbf{C ~ V}^{3}$ \\ ${ }^{1} M$ Tech, Student, SOE, CUSAT, Kerala, India \\ ${ }^{2} M$ Tech, Student, SOE, CUSAT, Kerala, India \\ ${ }^{3}$ Assistant Professor, SOE, CUSAT, Kerala, India
}

\begin{abstract}
In recent year, there has been high research attention in the field of user cooperation in wireless communication networks. Here a survey of cooperative diversity enabled medium access control protocols for WLANs are presented. Cooperative diversity is a promising technique which can combat fading and improve reliability in wireless environment. It developed as an alternative to MIMO systems where nearby terminals share their antennas and resources and hence create a Virtual MIMO (VMIMO) system. These terminals cooperate with the transmitter and receiver to transmit multiple copies of the same data to the receiver through independent fading channels. These multiple copies of the data can be combined at the receiver using appropriate techniques to recover the original packet correctly. This improves the reliability of the wireless channel by exploiting the spatial diversity. MAC protocols play an important role in realizing this concept by effectively coordinating transmissions between source, relay and destination nodes. For this reason, the research community has proposed a large number of cooperative MAC protocols to realize this concept. Most of these protocols are contention based and hence are based on IEEE 802.11 DCF mode. These protocols can be classified in to different categories based on condition for cooperation, optimal helper selection procedure, etc. The protocols discussed here belongs to a category in which the decision for cooperation based on a cooperation condition, preparation of potential helper lists and optimal helper selection are done by the source itself. An overview of these protocols including handshaking schemes, medium access schemes, and features are discussed.
\end{abstract}

Keywords: Cooperative Diversity, Medium Access Control, Virtual MIMO, Wireless LAN

\section{INTRODUCTION}

Cooperative diversity can be used to nullify the effects of signal fading due to multipath propagation in wireless networks. Cooperative diversity is a kind of space diversity which creates a "virtual array" from adjacent terminals by sharing their antennas and resources through transmission in distributed manner and signal processing [1], [2]. It is also termed as user cooperation diversity [3].

\subsection{Cooperation in WLAN}

Fig -1 shows an example wireless network. Let terminals $\mathrm{T} 1, \mathrm{~T} 2$ be the transmitters and $\mathrm{T} 3, \mathrm{~T} 4$ be the intended receivers. Consider the case $\mathrm{T} 3 \neq \mathrm{T} 4$. It corresponds to an ad hoc mode of operation in WLAN. Case T3=T4 correspond to an infrastructure mode of operation in WLAN and terminal T3 acts as an access point here [4]. Any terminal can receive and process the transmitted signal due to the broadcast nature of transmissions in a WLAN. Protocols used in WLAN often avoid such extra observations of transmitted signals. Making use of these signals, T1 and T2 can jointly communicate their information. They can share their resources like power, bandwidth, etc for transmission. Scenario $\mathrm{T} 3=\mathrm{T} 4$ can be considered as a wireless multiple access channel with relaying and scenario $\mathrm{T} 3 \neq \mathrm{T} 4$ can be treated as a wireless interference channel with relaying. To transmit the information of T1, T2 acts as a "relay" and share the resources with T1. Same method can be used for transmitting the information of $\mathrm{T} 2$.

$* * *$

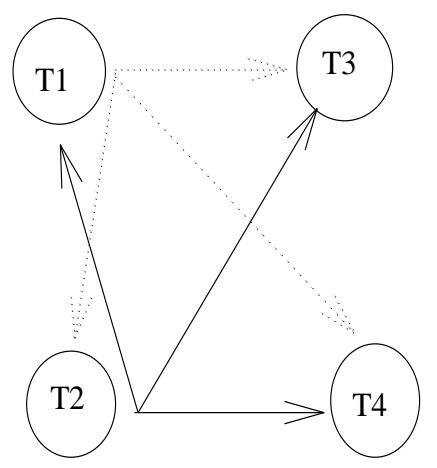

Fig -1: Cooperative diversity scenario [1]

\subsection{Cooperative Diversity Protocols}

Different cooperative diversity protocols with less complexity can be used in WLAN of Fig -1. Fixed, selection and incremental relaying [1], [2] protocols are some of them. Relay terminals uses different processing techniques and destination terminals uses different combining techniques based on these protocols. In fixed relaying, the relays are allowed to either amplify the received signals based on their power constraint, or to decode, encode, and retransmit the messages. Selection relaying is based on fixed relaying and the transmitting terminals can use cooperative or non cooperative transmission based on the measured SNR between the terminals. Incremental relaying makes use of the limited feedback obtained from the destination to decide whether relaying is necessary. It improves the spectral efficiency compared to fixed and selection relaying. 


\subsubsection{Fixed Relaying}

It is of two types: Amplify and Forward [1] and Decode and Forward [1]. In the first method, source terminal transmits data to the destination. During this time, relay processes the received signal from source and then relays the amplified signal to the destination. This method is same as repetition coding from two different transmitters except that the relay boosts its own receiver noise along with the signal. Suitable combining techniques are used for combining the signals from two sub blocks and then the information is decoded at the destination. In Decode and Forward method, the relay decodes the transmitted signal from source and processes it. It then transmits the signal though a repetition coding scheme. The relay can use different decoding techniques and different combining techniques can be used at the destination.

\subsubsection{Selection Relaying}

In this the path between source and relay is checked initially in terms of SNR. If the path is poor, the source continues the transmission to the destination either using repetition scheme or using more powerful coding techniques. If the path is good, relay adopts fixed relaying to achieve spatial diversity gain.

\subsubsection{Incremental Relaying}

Relay just repeats the transmission in both fixed and selection relaying. So, both the protocols do not make full use of degrees of freedom at higher rates. Incremental relaying is used to overcome this drawback. If the direct transmission is failure, a negative acknowledgement via feedback is provided by the destination. Then the relay retransmits the information and achieves spatial diversity. This kind of procedure improves the spectral efficiency of incremental relaying protocol compared to the previous ones. Incremental relaying protocol makes full use of degrees of freedom since it repeats the transmission only rarely.

Separate sub channel for each relay is required to repeat transmission in all the above methods. Also, as the number of cooperating terminals increases the bandwidth efficiency decreases in these methods. Coded cooperation [5] can be used to overcome these limitations and spatial diversity can be achieved. One of such method is space time coded cooperative diversity algorithms [6]. All relays can transmit on the same sub channel in these algorithms. So it improves bandwidth efficiency. The advantages of these algorithms also include full benefits of spatial diversity and non requirement of feedback. The main disadvantage is the increase in computational complexity.

Fig -2 illustrates the two transmission phases [1], [2] of the above said algorithms. During the first phase, source broadcasts the message and both destination and potential relays overhear the transmission. In the second phase, potential relays repeat the transmission to the destination. It is different in these two algorithms. In repetition based algorithm, each relay repeats the transmission in separate sub channel. In space time coded algorithm, each relay repeats the transmission in the same sub channel simultaneously.

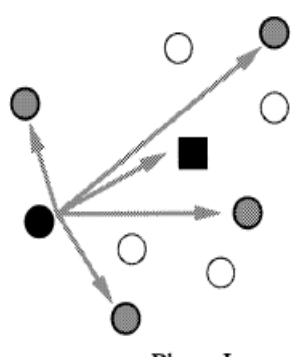

Phase I

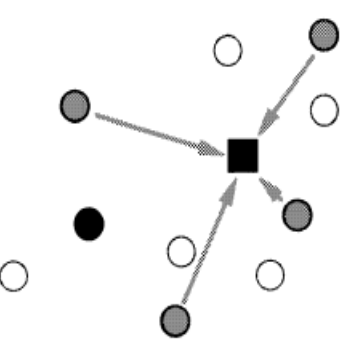

Phase II
Fig -2: Illustration of the phases of diversity algorithms [6]

Terminals transmit on orthogonal channels for medium access control. Fig -3 illustrates the channel allocation in the case of transmission without cooperation. Fig -4 illustrates the channel and sub channel allocations in the case of cooperative diversity algorithms based on repetition [1], [2]. These channels must be orthogonal so that at the receiver these signals can be combined and spatial diversity can be achieved. Fig -5 illustrates channel and sub channel allocations in the case of cooperative diversity algorithms based on space time coding. Here, space time codes are used by the relays in the second phase so that relays can repeat the transmission in the same channel simultaneously [6].

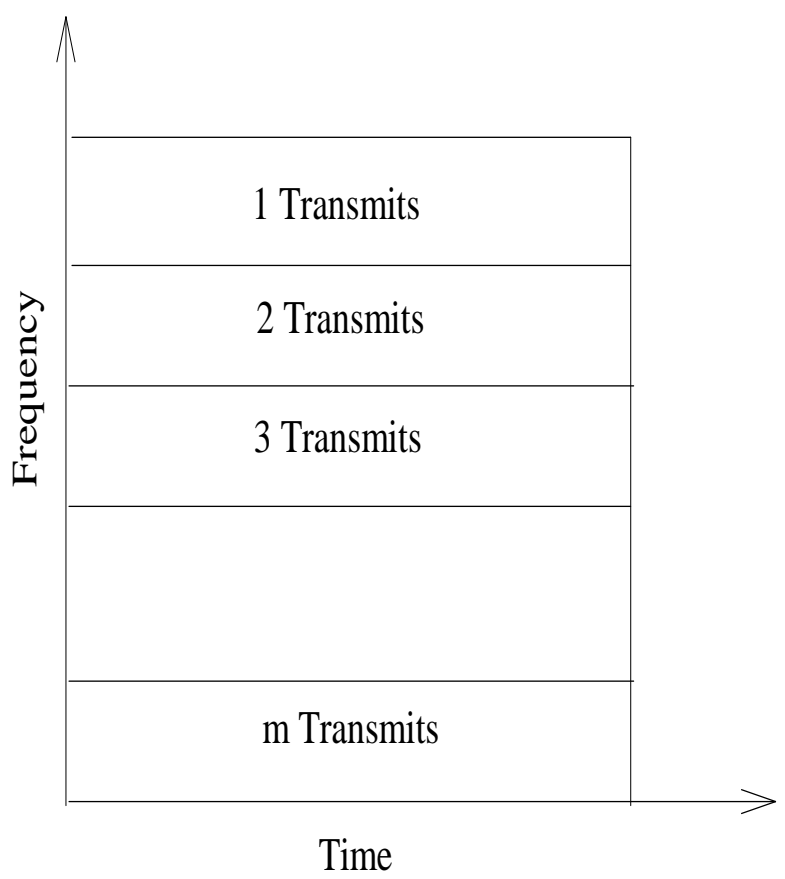

Fig -3: Medium access control without cooperation [6] 


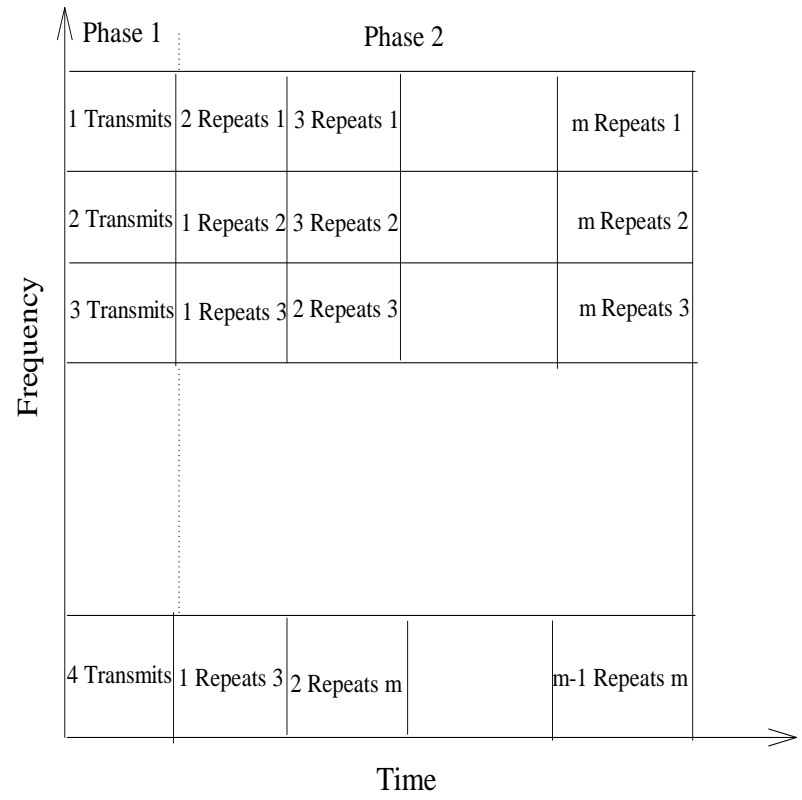

Fig -4: Medium access control based on repetition [6]

\begin{tabular}{|c|c|}
\hline \multicolumn{1}{|c|}{ Phase 1 } & Phase 2 \\
\hline 1 Transmits & $\mathrm{D}(1)$ Relay \\
\hline$\underset{\circlearrowright}{\circlearrowright}$ & $\mathrm{D}(2)$ Relay \\
\hline 2 Transmits & $\mathrm{D}(3)$ Relay \\
\hline 3 Transmits & \\
\hline & \\
\hline $\mathrm{m}$ Transmits & $\mathrm{D}(\mathrm{m})$ Relay \\
\hline
\end{tabular}

Time

Fig -5: Medium access control by space time coding [6]

\subsection{Medium Access Control in WLANs}

WLANs are specified by the IEEE 802.11 standard [7]. The architecture of the MAC sub layer, as in Fig -6, consists the distributed coordination function (DCF), the point coordination function (PCF), the hybrid coordination function $(\mathrm{HCF})$ and the mesh coordination function (MCF) [7].

Normally MAC protocols can be divided in to two: channel allocation based (or contention free) MAC and contention based random access MAC. Both the MACs can be used in a cooperative scenario with some modifications. Contention based MAC is described here since more researches are conducting on this. Contention based MAC protocols often use contention based mode called DCF shown in Fig -7. Distributed Coordination Function is basically a Carrier Sense Multiple Access scheme with Collision Avoidance (CSMA/CA) [7], where a station is allowed to transmit if the medium is sensed to be idle (i.e., no other station is transmitting). Request To Send (RTS) and Clear To Send (CTS) [7] control frames are used to inform channel reservation time and prevent neighbouring nodes from simultaneously transmitting, as shown in Fig -7. Here, DIFS (Distributed Inter Frame Space) and SIFS (Short Inter Frame Space) [7] are used for frame spacing. Frame spacing allows assigning priorities to a transmission based on their type (control or data frame). The control frames (RTS/CTS) include the addresses of both source and destination and a Network Allocation Vector (NAV) which specifies the time duration of busy medium condition.

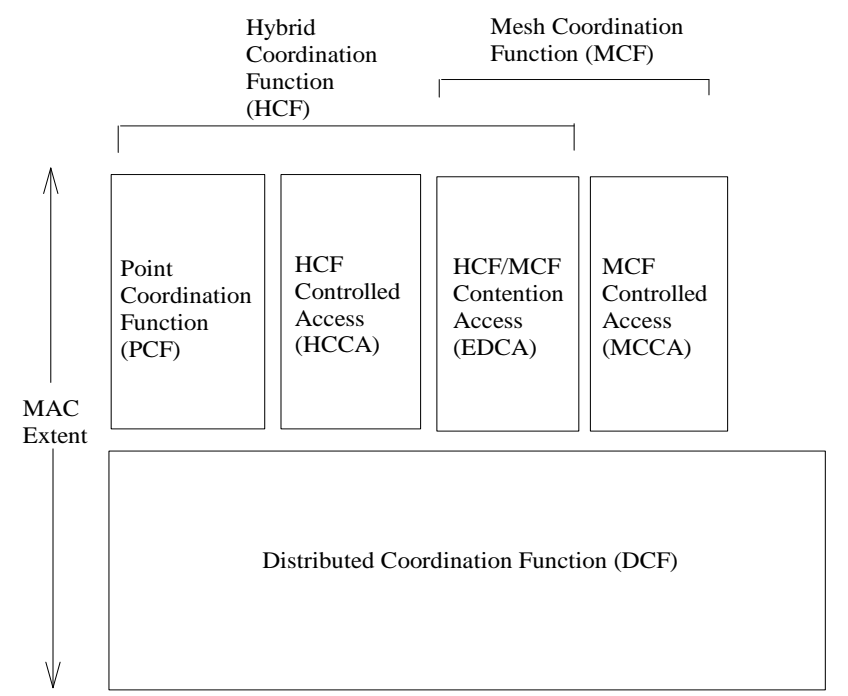

Fig -6: MAC architecture [7]

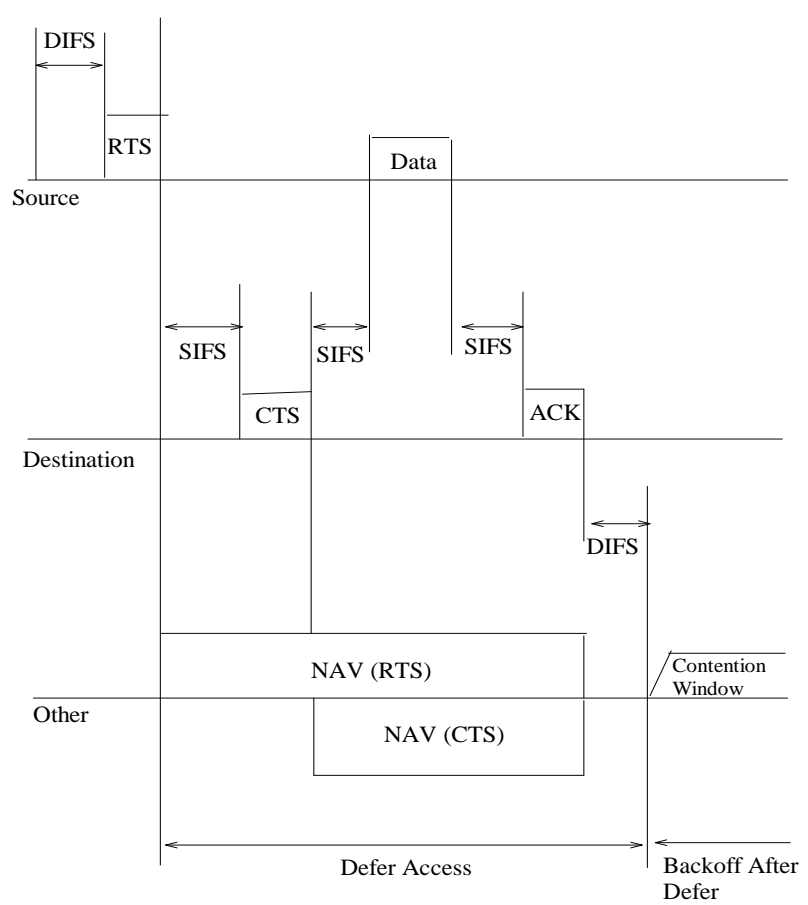

Fig -7: Distributed Coordination Function [7]

While most of this initial work focused on physical layer schemes [1], [2], [6], [8], [9] researchers have also investigated supporting cooperative diversity in higher layer protocols, mostly in the MAC layer. The initial attention was focused on developing cooperative MAC protocols for 
WLANs based on the IEEE 802.11 standard [7]. Here, the advances made in developing cooperative MAC protocols for WLANs are presented. An overview of their functionality is discussed here. Also results and observed shortcomings are discussed.

\section{COOPERATIVE MAC PROTOCOLS FOR}

\section{WLAN}

\subsection{CoopMAC: A Cooperative MAC for WLANs}

CoopMAC [10] illustrates how the IEEE802.11 distributed coordination function (DCF) [7] can be modified to use cooperative communication for achieving both higher throughput and lower interference. In CoopMAC, nodes have the option of switching between direct and cooperative transmission. When using cooperative transmission, a sender communicating with a receiver at a low data rate can ask a neighboring helper, with a higher data rate with both itself and the destination, for cooperation by forwarding its packets. CoopTable, shown in Table -1 , maintains the information of potential helper nodes from which the optimal helper node is selected. Alternatively, when using the infrastructure mode, the Access Point (AP) can also maintain such a CoopTable for each node, while individual nodes only need to keep track of the AP.

Table -1: Format of the CoopTable [10]

\begin{tabular}{|c|c|c|c|c|}
\hline ID (48 bits) & ) Time ( 8 bits) & $\mathrm{R}_{\mathrm{hd}}(8$ bits $)$ & $\mathrm{R}_{\mathrm{sd}}(8$ bits $)$ & Number of Failures \\
\hline$\ldots \ldots \ldots$ & $\ldots \ldots \ldots . .$. & $\ldots \ldots \ldots \ldots$ & ................... & ․․․․․․․․․…… \\
\hline
\end{tabular}

CoopTable is filled with appropriate data by a node by overhearing the ongoing transmissions. Consider a network with a source $S_{\mathrm{s}}$, helper $S_{\mathrm{h}}$, destination $S_{\mathrm{d}}$. It can be assumed that the source knows the data rate between itself and $D, R_{s d}$, from previous transmissions. Source overhears and analyzes the transmission (including RTS/CTS packets and data packets) between $\mathrm{H}$ and $\mathrm{D}$ to decide whether neighbouring node $\mathrm{H}$ can cooperate with it. The data rate between $\mathrm{H}$ and $\mathrm{D}, \mathrm{R}_{\mathrm{hd}}$, is obtained from the Physical Layer Convergence Procedure (PLCP) header [10]. CoopTable stores all these information along with time stamps and any consecutive failed cooperation attempt by $S_{h}$.

When $S_{\mathrm{s}}$ want to transmit data to $S_{d}$, it compares the amount of time required to transmit via direct link and helper node. It simply involves the adding up the payload size, header size, control packet size and then dividing it with the transmission rate of the corresponding links. All these required information are stored in the CoopTable. If the indirect link through $\mathrm{S}_{\mathrm{h}}$ is more efficient than the direct link, CoopMAC goes for cooperation. The first step for cooperation is cooperative handshaking which is shown in Fig -8. For this, an additional frame HTS (Helper Ready to
Send) [10] is introduced. The source puts the address of both the helper and the destination in the RTS frame along with the request to cooperate. The helper, if available, acknowledges this with an HTS. Finally, the destination responds with a CTS followed by the transfer of data as shown in Fig -9.

The main feature of CoopMAC is that it is backward compatible with the existing IEEE 802.11 standard [7]. A drawback of CoopMAC is that it either uses the direct source to destination channel or the indirect source to relay to destination channel. Since only one packet is received at the destination, it cannot use a packed combining scheme and take advantage of redundancy in the form of a repeated packet. Therefore, CoopMAC, in a strict sense, is not a cooperative protocol as its diversity degree is only one. It is selective in practice. It is shown that CoopMAC can provide significant throughput and delay improvements, without much complexity in system design.

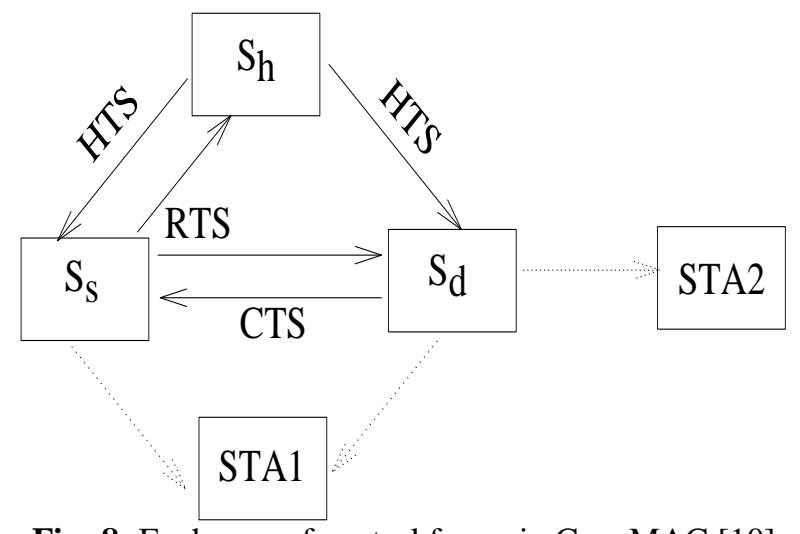

Fig -8: Exchange of control frame in CoopMAC [10]

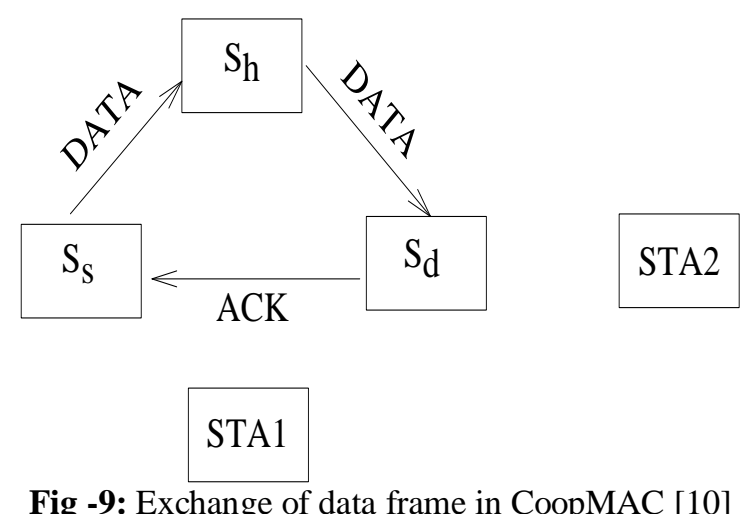

\subsection{C-MAC}

C-MAC [11] is comparable to IEEE 802.11 [7] in many aspects. It is developed based on DCF mode [7] of IEEE 802.11. C-MAC without relay is same as that of IEEE 802.11.C-MAC with relay, as shown in Fig -10, have some modifications. The main difference is in the handshake procedure. Control frames include CoopRTS/RTR/CTS instead of RTS/CTS used in IEEE 802.11. RTS frame is modified to CoopRTS with some additional features. RTR (Ready to Relay) frame is used by the relay to announce that it is ready for cooperation. RTR frame can be decoded by 
the MAC layer since its frame format is similar to that of CTS. Based on the prior transmission knowledge, source (S) chooses either relay mode or regular mode for data transmission. CoopRTS/RTR/CTS handshake procedure is used to ensure the participation of relay node in the transmission procedure. In C-MAC with relay, data transmission starts when the relay node $(\mathrm{R})$ is ready to help. Source (S) just broadcasts the packet intended for destination (D). Relay node can overhear this transmission. Destination (D) keeps the received signal from source (S) and wait for the signal from relay node (R). Relay transmits the overheard signal from source to destination after a SIFS time. Joint decoding of these two signals is performed at the destination physical layer and a frame is sent to the MAC layer. If the Frame Check Sequence (FCS) is correct, destination responds with a positive ACK feedback.

C-MAC protocol is backward compatible with IEEE 802.11. It is not a perfect one since it requires a method to find potential relays. It requires physical layer support for cooperation to jointly decode the received signals at the destination. But, it provides diversity since both the direct and indirect link (if available) is used for transmission [12].
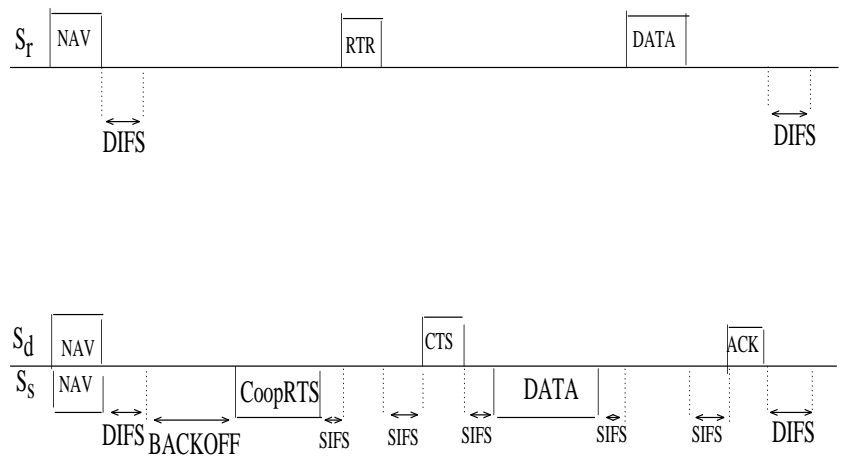

\begin{tabular}{|c|c|c|}
\hline \begin{tabular}{ll|} 
STA2 & NAV \\
\end{tabular} & & NAV (CTS) \\
\hline STA1 ${ }_{\text {NAV }}$ & & NAV (CoopRTS) \\
\hline
\end{tabular}

Fig -10: C-MAC with relay [11]

\subsection{CD-MAC}

Cooperative Diversity MAC (CD-MAC) [13] is a protocol which is useful ad hoc networks. This protocol uses spacetime-coded cooperative diversity algorithm. Distributed Space Time Code (DSTC) is used in this protocol and hence all the relays can transmit on the same channel simultaneously [14]. This protocol works on a single channel and uses only one relay for a particular retransmission. CD-MAC is developed based on DCF mode of IEEE 802.11 standard and hence it is backward compatible.
CD-MAC uses a four-way handshaking procedure for cooperation as shown in Fig -11. If direct link is reliable, conventional MAC (DCF) is used, otherwise cooperation is preferred. Source node sends a RTS to the destination. If the destination replies with CTS, then the succeeding data transfer is done without cooperation. However, if the source fails to receive CTS from the destination node and the timeout period expires, it activates cooperation in the second phase. The source informs this to the receiving node by using a relay address in the repeated RTS packet. During cooperation, the source and relay both use DSTC. In the first phase, source just sends the packet to the relay. In the second phase, both relay and source transmits the DSTC coded copies of the same packet to the destination simultaneously. In Fig -11, C-RTS represents coded RTS, which means DSTC is also utilized for control packets. When the destination wants to reply to a C-RTS, it also selects a relay and sends C-CTS simultaneously with the relay. Following this, data transmission and ACK packets are transmitted. Since, both the links are used for transmission, this protocol provides diversity.

\begin{tabular}{|c|c|c|}
\hline \multicolumn{2}{|c|}{ Timeout } & SIFS \\
\hline Sender RTS & \begin{tabular}{|l|l|} 
RTS & C-RTS \\
\end{tabular} & DATA $\mid$ C-DATA \\
\hline
\end{tabular}

Relayl

$$
\text { Relay2 }
$$$$
\text { C-CTS }
$$
C-ACK

Fig -11: CD-MAC four way handshaking [13]

\section{CONCLUSIONS}

Some of the MAC protocols for cooperation in WLANs are analyzed. It is clear that protocols for WLANs have reached a certain maturity, owing to significant interest of the research community, and some protocols are quiet feasible for deployment on existing hardware, such as CoopMAC. Almost all of the proposed protocols are contention-based and use a modified version of 802.11 DCF mode for cooperative handshaking.

All the above protocols fall below a category of protocols in which the decision for cooperation based on a cooperation condition, preparation of potential helper lists and optimal helper selection are done by the source itself. There are other categories of protocols in which the above functions are shared by helper node and source. Some of them are: rDCF [15], ErDCF [16] in which preparation of potential list of helper nodes are done by helper contention, Feeney-MAC [17], Shan-MAC [18] in which optimal helper itself is selected by helper contention, etc. But the category of protocols which has got research attention is described here including CoopMAC, C-MAC, and CD-MAC and other 
ones like Ahmed MAC [19], ADC-MAC [20]. The main reasons include the facts that these protocols are straight forward, they can be easily implemented by simply extending the existing IEEE 802.11 MAC and they are similar to the physical layer cooperative diversity protocols [1], [2], [6], [8], [9]. But this cooperation introduces large overhead for source since it has to maintain information related to all the potential relays (e.g. CoopTable in [10]). Also it is difficult to maintain accurate information about the nodes with mobility or nodes in a rapidly changing environment. Efficient algorithms for choosing optimal helper from potential helper nodes are also necessary.

It can be seen that a lot of excellent research on cooperation in WLAN have conducted already. But, some unaddressed issues still remain in this area. Many of the protocol discussed here chooses only one relay node. But protocols using multiple helper nodes can bring the MIMO benefits well and can fully exploit spatial diversity. There is no such protocol in the literature yet which can be pointed out. Mobility, Security and energy consumption are still relevant research problems in the cooperative scenario. All these issues have to be solved properly so that a high performance MAC with cooperation can be implemented in practice.

\section{REFERENCES}

[1]. J. Laneman, D. Tse, and G. Wornell, "Cooperative Diversity in Wireless Networks: Efficient Protocols and Outage Behavior", IEEE Trans. Inf. Theory, vol. 50, pp. 3062-3080, Dec. 2004.

[2]. J. N. Laneman, G. W. Wornell, and D. N. C. Tse, “An efficient protocol for realizing cooperative diversity in wireless networks," in Proc. IEEE Int. Symp. Information Theory, Washington, DC, June 2001.

[3]. A. Sendonaris, E. Erkip, and B. Aazhang, "Increasing uplink capacity via user cooperation diversity," in Proc. IEEE Int. Symp. Information Theory (ISIT), Cambridge, MA, Aug. 1998, p. 156.

[4]. V. Hayes, IEEE Standard for Wireless LAN Medium Access Control (MAC) and Physical Layer (PHY) Specifications, 1997.

[5]. Hunter, T.E., Nosratinia, A.: 'Diversity through coded cooperation', IEEE Trans. Wirel. Commun., 2006, 5, (2), pp. 283-289.

[6]. J. Laneman and G. Wornell, "Distributed space-time coded protocols for exploiting cooperative diversity in wireless networks," IEEE Transactions on Information Theory, Vol. 49, No. 10, October 2003.

[7]. L. standards Committee, "Part 11: Wireless LAN medium access control (MAC) and physical layer (PHY) specifications", IEEE-SA Standards Board, 2003.

[8]. A. Sendonaris, E. Erkip, and B. Aazhang, "User cooperation diversity part I: system description", IEEE Trans. Commun., vol. 51, pp. 1927-1938, Nov. 2003.

[9]. A. Sendonaris, E. Erkip, and B. Aazhang, "User cooperation diversity part II: implementation aspects and performance analysis", IEEE Trans. Commun., vol. 51, pp. 1939-1948, Nov. 2003.
[10]. Liu, P., Tao, Z., Narayanan, S., Korakis, T., Panwar, S.S.: 'CoopMAC: a cooperative MAC for wireless LANs', IEEE J. Sel. Areas Commun., 2007, 25, (191), pp. 340-354. [11]. H. Jin, X. Wang, H. Yu, Y. Xu, Y. Guan, and X. Gao, "C-MAC:A MAC Protocol Supporting Cooperation in Wireless LANs",2009 IEEE Wireless Communications and Networking Conference, pp. 1-6, Apr. 2009.

[12]. P. Ju, W.Song, D. Zhou, 'Survey on cooperative medium access control protocols', IET Commun., 2013, Vol. 7, Iss. 9, pp. 893-902.

[13]. Sangman, M., Chansu, Y., Seung-Min, P., HeungNam, K., Jiwon, P.: 'CD-MAC: cooperative diversity MAC for robust communication in wireless ad hoc networks', Proc. IEEE ICC, 2007, pp. 3636-3641.

[14]. G. Scutari and S. Barbarossa, "Distributed Space-Time Coding for Regenerative Relay Networks", IEEE Trans. On Wireless Communications, Vol. 4, No. 5, pp. 2387-2399, Sep. 2005.

[15]. Zhu, H., Cao, G.: 'rDCF: A relay-enabled medium access control protocol for wireless ad hoc networks', IEEE Trans. Mob. Comput, 2006, 5, (9), pp. 1201-1214.

[16]. Ahmad, R., Zheng, F., Drieberg, M., Olafsson, S.: 'An enhanced relay enabled medium access control protocol for wireless ad hoc networks'.Proc. IEEE VTC, Spring, 2006.

[17]. Feeney, L.M., Cetin, B., Hollos, D., Kubisch, M., Mengesha, S., Karl, H.: 'Multi-rate relaying for performance improvement in IEEE 802.11 WLANs', Proc. Fifth Int. Conf. on Wired/Wireless Internet Communications (WWIC), 2007.

[18]. Shan, H., Cheng, H., Zhuang, W.: 'Cross- layer cooperative MAC protocol in distributed wireless networks', IEEE Wireless Commun., 2011, 10, (8), pp. 2603-2615.

[19]. Ibrahim, A.S., Sadek, A.K., Su, W., Liu, K.J.R.: 'Cooperative communications with relay-selection: When to cooperate and whom to cooperate with?' IEEE Trans. Wirel.Commun., 2008, 7, (7), pp. 2814-2827.

[20]. Zhou, T., Sharif, H., Hempel, M., Mahasukhon, P., Wang, W., Ma, T.: 'A novel adaptive distributed cooperative relaying MAC protocol for vehicular networks', IEEE J. Sel. Areas Commun., 2011, 29, (1), pp. 72-82 .

\section{BIOGRAPHIES}

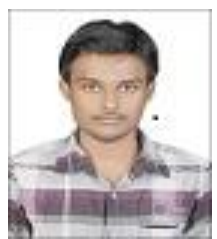

Unnikrishnan $M$ received the $B$ Tech Degree in Electronics and Communication Engineering from College of Engineering Trikaripur, CUSAT, Kerala, India, in 2012. $\mathrm{He}$ is now pursuing $\mathrm{M}$ Tech degree in Electronics and Communication with specialization in Wireless Technology at CUSAT, Kochi, India. E-Mail: unnikrishnanm60@gmail.com

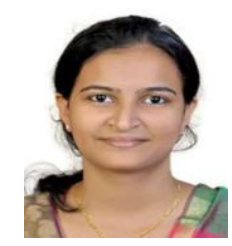

Rinu Titus received her B.Tech degree from Sahrdaya College of Engineering and Technology, University of Calicut in 2012. She is currently pursuing her M.Tech degree in Wireless Technology from School of Engineering, CUSAT.

E-Mail: rinutitus@gmail.com 
Premkumar C V received his M.Tech from CUSAT in 2005 June. He is currently serving as a faculty member at CUSAT since 2001. His interested research field is Wireless Communication.

E-Mail: premkumar@cusat.ac.in 